FSS

Zhao, P., \& Lapierre, M. A. (2020). Stress, dependency, and depression: An examination of the reinforcement effects of problematic smartphone use on perceived stress and later depression. Cyberpsychology: Journal of Psychosocial Research on Cyberspace, 14(4), article 3. https://doi.org/10.5817/CP2020-4-3

\title{
Stress, Dependency, and Depression: An Examination of the Reinforcement Effects of Problematic Smartphone Use on Perceived Stress and Later Depression
}

\author{
Pengfei Zhao \& Matthew A. Lapierre \\ Department of Communication, University of Arizona, Tucson, Arizona, USA
}

\begin{abstract}
Problematic Smartphone Use (PSU) is associated with a series of mental health issues including depression; yet, the directionality and underlying mechanisms for these associations are underdetermined. This study investigated the possible reinforcement effects of PSU on perceived stress and later depression and the moderating effect of social support on the associations between perceived stress and smartphone use and PSU. This study further explored the differential effects for separate types of smartphone use. With a sample of 222 college students, we found that PSU reinforced perceived stress especially for people with high levels of stress, and perceived stress fully mediated the association between PSU and depression. Regarding types of smartphone use, we found that perceived stress was associated with both person-to-person and social networking smartphone use. In addition, for people with low to medium levels of social support, perceived stress was linked to increased PSU via social networking smartphone use.
\end{abstract}

Keywords: Problematic smartphone use; smartphone use; perceived stress; depression; social support

\section{Introduction}

The smartphone has become an essential communication and entertainment device over the last decade. According to Taylor \& Silver (2019), 81\% of American adults own a smartphone while 95\% of young adults (aged 18 to 34) report having a smartphone. Considering the rates of smartphone ownership, increased scholarly attention has been paid to understanding how smartphones might affect users (Elhai, Dvorak, et al., 2017). In particular, a substantial body of research has suggested that problematic smartphone use (Billieux, 2012) is associated with a series of negative outcomes, such as sleep disturbances, poor academic performance, vocational impairment, as well as mental health problems including depression, loneliness, and anxiety (Demirci et al., 2015; Duke \& Montag, 2017; Gutiérrez et al., 2016; Samaha \& Hawi, 2016). Note that, although problematic internet use has been widely studied (Li et al., 2010; Yao \& Zhong, 2014), PSU may generate additional risks and requires further examination (Gutiérrez et al., 2016). Problematic internet use is usually linked with general computer use and includes problematic use of internet chats, games, and information seeking (Elhai, Dvorak, et al., 2017). Although smartphones offer some similar affordances with the internet via computer use, smartphones are involved in daily lives in qualitatively different ways as smartphones unique functions and affordances, such as portability, wireless data access, and a greater variety of applications dwarfs computer use (Lapierre et al., 2019).

While PSU has been linked to the above outcomes, the directionality and underlying mechanisms associated with these harms are underdetermined. First, it is unclear whether PSU produces these outcomes or if things like sleep 
issues and depression actually produce increased PSU. Second, even if one finds that PSU produces these outcomes, it is important to understand how it has these effects. Third, understanding the risk factors of PSU can be helpful for reducing dependence on smartphones and corresponding detrimental outcomes. To that end, rooted in the stress-coping framework and previous literature (Lazarus \& Folkman, 1984; Pivetta et al., 2019), the current study uses a two wave design to help determine the serial ordering of perceived stress, PSU, and depression, and also investigates whether perceived stress provides some explanatory power for why the link between PSU and depression exists. Specifically, this study investigates whether there is a reinforcement effect of PSU on perceived stress. In other words, we examine whether perceived stress leads to later PSU, which, in turn, amplifies perceived stress. Exploring this reinforcement effect can help researchers and practitioners understand the downward spiral or vicious circle between perceived stress and PSU, which can also contribute to understanding the negative role that PSU may play in shaping personal well-being.

Furthermore, it is important to also examine what factors can serve to protect smartphone users from problematic use, stress, and depression. To wit, based on the buffering model (Cohen \& Wills, 1985), social support has been demonstrated to reduce physiological and psychological stress appraisals and protects individuals from the negative effects that stress has on well-being. This study explores whether social support moderates the effect of perceived stress on both smartphone use and PSU. Lastly, it is necessary to understand the role that smartphone use plays. Most of the research on the effects of smartphone use have looked at this device in a monolithic fashion (Rotondi et al., 2017) but it seems more appropriate to look at use via the types of use that are available (Elhai, Levine, et al., 2017). Therefore, this study explores the relationships among differing types of smartphone use (i.e., person-to-person use, mass mediated use, and social networking use) on users.

\section{The Reinforcement of PSU on Perceived Stress and Later Depression: A Stress-Coping Approach}

The stress-coping framework argues that people often engage in maladaptive coping strategies when experiencing stressful events (Lazarus \& Folkman, 1984). Stress develops when people have a perceived inability to cope with demands and threats made on them (Lazarus, 1966). Biological, psychological, sociological, as well as philosophical stressors can cause stress (Greenberg, 1981). Strategies to cope with stress were broadly grouped into two categories: problem-focused and emotion-focused (Lazarus \& Folkman, 1984). Problem-focused coping strategies aiming at "resolving the stressful situation or event or altering the source of the stress" (Carroll, 2013, pp. 1540-1541) are considered as adaptive. However, emotion-focused coping strategies focusing on the regulation of stressful emotions are considered as maladaptive (Fryer et al., 1997; Mayhew \& Edelmann, 1989). Research has shown that compared to emotion-focused coping strategies, problem-focused strategies are more likely to cause positive outcomes as emotion-focused coping strategies are unable to deal with the source resulting in stress (i.e., Lewin \& Sager, 2008). When preferring emotion-focused or maladaptive coping strategies, perceived stress can result in unhealthy behaviors such as problematic eating and substance use (e.g., Wichianson et al., 2009).

Some typical maladaptive coping strategies include avoidance, self-distraction, denial, and detachment (Folkman \& Lazarus, 1980). When preferring these strategies, media can be popular tools to avoid and escape from stressors (e.g., Beranuy et al., 2013). A considerable number of studies have found that people use digital media such as smartphones and social media to manage stress (George et al., 2013; Jun \& Choi, 2015). Specifically, KardefeltWinther (2014a, 2014b) framed problematic internet use as a dysfunctional coping strategy to escape real life issues, which is related to negative outcomes. In addition, a theoretical model (i.e., the Interaction of PersonCognition-Execution Model; I-PACE) was proposed recently to explain the factors and underlying mechanisms, such as stress and coping, resulting in problematic internet use (Brand et al., 2016). Specifically, the I-PACE model suggests that when people prefer to maladaptive coping strategies when being confronted with a stressful situation, they are likely to use internet to regulate their mood if they consider internet use as stress-relieving, which lays foundation for the later problematic internet use. Based on the stress-coping framework and other models addressing problematic technology use such as the I-PACE model, we also assume that PSU is a representation of maladaptive coping strategies as it cannot help dealing with the source of stress but is for escaping from stressful events and regulating one's mood, and related to detrimental outcomes such as decreased well-being. 
PSU refers to an excessive or uncontrolled use of one's smartphone (Billieux, 2012), with labels varying from PSU, to smartphone addiction, to smartphone dependency. This phenomenon is characterized by loss of control, tolerance, withdrawal symptoms, and functional impairment (Billieux, 2012; D. Kim et al., 2014). Although PSU is not included in Diagnostic and Statistical Manual for Mental Disorders (DSM-5; American Psychiatric Association, 2013), researchers have suggested that PSU should be considered a behavioral addiction as it functions similarly to substance addictions such as alcohol and drug addiction (Billieux, 2012; Kwon et al., 2013). In addition, internet gaming addiction has been described in DSM-5 as a note-worthy condition requiring more clinical research before being evaluated as an official disorder, which may mean that digital addictions, such as PSU, have important clinical implications like other addictive disorders that require further examinations.

Extant studies have found that PSU is associated with sleep disturbances (Demirci et al., 2015), poor academic performance (Samaha \& Hawi, 2016), loss of productivity (Duke \& Montag, 2017), as well as mental health problems including depression, loneliness, and anxiety (Elhai, Dvorak, et al., 2017). Considering the number of problematic outcomes associated with PSU, it is essential to examine the risk factors of PSU as understanding the risk factors can be helpful to effectively reduce PSU and corresponding detrimental outcomes. Also, given the negative effects of PSU on well-being, it is important to investigate the role that problematic use plays in the maintenance and development of mental health. Previous research has confirmed that perceived stress or stressful events were associated with internet or PSU (Elhai, Dvorak, et al., 2017; Jun \& Choi, 2015). For example, stressful life events such as academic underachievement and deviant peer affiliation can cause problematic internet use (Jun \& Choi, 2015; Li et al., 2013). Furthermore, Samaha and Hawi (2016) found that PSU was positively associated with perceived stress, and the latter was negatively associated with life satisfaction. Therefore, based on the stress-coping framework and previous literature, the current study hypothesizes:

\section{H1: Perceived stress is positively associated with later PSU.}

However, PSU may not help individuals cope with their stressors effectively; contrarily, PSU can cause a number of negative psychosocial outcomes (Gutiérrez et al., 2016). For example, PSU could lead to increased withdrawal from others or an inability to accomplish work/academic tasks (Tokunaga, 2014). These negative outcomes associated with PSU may further reduce people's perceived control over their life circumstances, and thus reinforce their perceived stress. Therefore, this study hypothesizes:

\section{H2: PSU is positively associated with perceived stress.}

As Billieux (2012) suggested, psychological factors such as personality traits, impulsivity facets, and self-esteem can lead to PSU, which, in turn, results in negative outcomes including reduced psychological well-being. Among the problematic outcomes, depression is of primary concern because it is associated with an increased prevalence of other chronic diseases (Chapman et al., 2005) and even suicide (Kandel et al., 1991). Extant cross-sectional research has found there is a well-established relationship between PSU and depression (Aker et al., 2017; Alhassan et al., 2018). More importantly, a recent longitudinal study has found that PSU predicted increased depressive symptoms over time rather than the opposite (Lapierre et al., 2019). Supporting this finding, a fivewave longitudinal study on problematic internet use also revealed that, among adolescents, compulsive internet use (i.e., being incapable of regulating one's internet use), predicted later depression (Muusses et al., 2014). Therefore, based on the previous research, the current study hypothesizes:

\section{H3: PSU is positively associated with depression.}

Stress is also consistently linked with depression (Hammen, 2005). For example, based on a sample of 500 adolescents aged from 14 to 17, Dhillon and Arora (2017) found that perceived stress is a predictor of depression. Stress may lead to depression via brain disturbances. Specifically, Van Praag (2004) suggested that the changes in 5-hydroxytryptamine and stress hormone caused by stressors are similar to the disturbance in these systems observed in depression. In other words, perceived stress may cause brain dysfunctions, and brain dysfunctions are a risk factor for depression. Given the consistent link between stress and depression, this study hypothesizes:

H4: Perceived stress is positively associated with depression. 
In fact, it may be the case that perceived stress mediates the relationship between PSU and depression. As noted above, due to the negative outcomes caused by PSU, PSU might reinforce perceived stress, and increased perceived stress can lead to depression. Therefore, the current study hypothesizes that perceived stress may be a mediator of the association between PSU and depression.

H5: Perceived stress mediates the association between PSU and depression.

\section{The Buffering Effect of Social Support}

Social support has been demonstrated to reduce physiological and psychological stress appraisals and protects individuals from the negative impact that stress has on well-being (Cohen \& Wills, 1985). Cohen and Wills have suggested that social support buffers the negative effects of stress through two pathways. One pathway is that social support may prevent stress appraisal responses. Specifically, social support may offer necessary resources for people to cope with stressors; consequently, increased perceived ability to cope with stressors may prevent people considering a situation as stressful. The other hypothesized pathway is that when people appraise situations as stressful and thus experience great stress, social support can moderate the association between perceived stress and consequent maladjustive responses such as addictive behaviors.

Researchers have provided a substantial body of empirical support to the buffering model of social support, and these findings suggested that social support can reduce stress reactivity and further decrease negative behavioral and psychobiological outcomes of stress (e.g., Ditzen \& Heinrichs, 2014; Takizawa et al., 2006). In addition, previous studies have also suggested that social support can reduce individuals' addictive behaviors or can be beneficial for rehabilitation from addictive disorders. For example, Dobkin and colleagues (2002) have found that functional social support longitudinally predicts reductions in the severity of alcohol abuse. In addition, a variety of studies have found that perceived social support was negatively associated with internet addiction. For example, based on a sample of 1360 college freshmen, the findings showed that lower levels of social support was linked to higher rates of internet addiction (Tsai et al., 2009).

As noted above, perceived stress may predict later PSU as people with high perceived stress might be vulnerable for addictive disorders. However, according to the stress-buffering model of social support, increased social support may prevent people from experiencing the negative behavioral and psychobiological outcomes of perceived stress. Therefore, this study hypothesizes that social support is a moderator of the association between perceived stress and PSU.

H6: Social support moderates the association between perceived stress and PSU. The association between perceived stress and PSU will be stronger when people have low levels of social support comparted to when they have high levels of social support.

\section{Smartphone Use}

The role that smartphone use plays among these set of variables is also necessary to consider. Moreover, it is important to recognize that smartphones are multi-faceted devices which offer users an array of tools to connect with others. First, individuals can use smartphones to interact at the interpersonal level through using a series of tools such as text, email, and telephone calls. Second, a smartphone can exist as a mass media device as it allows people to read the news, surf the internet, watch videos, and play online games. Lastly, social networking sites (SNSs) such as Facebook and Instagram offer multiple affordances including making public posts and obtaining information; consequently, a smartphone, as a social networking device, functions between person-to-person communication and mass communication.

There is evidence that these separate types of smartphone use do affect users in different ways. For example, in a longitudinal study of teenagers, Bae (2019) found that increased smartphone use for interpersonal communication was associated with greater social capital over time, which, in turn, was associated with greater subjective well-being over time. Regarding social media use, Kim and colleagues (Y. Kim et al., 2016) found that social media use via smartphones facilitated college students' social engagement. In addition, other studies also suggested that smartphone use for communication was associated with increased social support and decreased 
Ioneliness/isolation (e.g., Cho, 2015). Therefore, it is essential to explore the effects associated with separate types of smartphone use.

Although smartphone use, especially use for communicative purposes, may be rewarding, the social benefits and pleasurable experiences that people obtain from smartphone use may lead to PSU. In a national survey study with 2212 Korean middle and high school students, Bae (2017) found that the frequency of both weekday and weekend smartphone use was associated with increased PSU. In particular, the amount of smartphone use for information seeking, entertainment seeking, and gaming was positively associated with PSU. In addition, Van Deursen and colleagues (Van Deursen et al., 2015) suggested that habitual smartphone use was an important predictor of PSU. Through examining the relationships between types of smartphone use and PSU, the authors found that processoriented smartphone use (i.e., content-based consumption of media) was positively associated with both habitual and addictive smartphone behaviors, and socially-oriented use of smartphone (i.e., use of social networking sites or instant messaging for social purpose) was positively associated with smartphone addiction via habitual smartphone use. Given the relationships between different types of smartphone use and PSU, this study hypothesizes:

H7a-c: Smartphone use (mass media, person-to-person, and social networking) is positively associated with later PSU.

Regarding the relationship between smartphone use and perceived stress, as an extension of use and gratification theory, compensatory internet use theory (CIUT) proposes that negative life events or stressors motivate people to use or excessively use technology for alleviating negative emotions and stress (Kardefelt-Winther, 2014a). Specifically, CIUT explains how social and psychological factors cause use/overuse of new technology. KardefeltWinther (2014b) examined the interaction effects of psychosocial variables (i.e., stress and self-esteem) and motivation of escapism on excessive online gaming. This study found that the interaction between stress and the motivation of escapism contributes to excessive online gaming. Similarly, based on CIUT, a great number of studies have suggested that stressful life events or perceived stress were associated with internet and smartphone use (e.g., Chiu, 2014). Therefore, the current study tests the relationships between perceived stress and types of smartphone use.

H8a-c: Perceived stress is positively associated with smartphone use (mass media, person-to-person, and social networking).

As mentioned above, perceived stress may lead to smartphone use, and smartphone use may cause later PSU; consequently, this study hypothesizes that smartphone use may be a mediator of the relationship between perceived stress and PSU.

H9a-c: Smartphone use (mass media, person-to-person, and social networking) mediates the association between perceived stress and PSU.

As previously mentioned, the stress-buffering model of social support assumes that social support can reduce the maladjustive and negative responses to perceived stress (Cohen \& Wills, 1985). Therefore, it is plausible that people with high levels of social support feel less compelled to use their smartphones to alleviate stress and negative emotions compared to individuals with low levels of social support. Under this condition, this study hypothesizes that social support may be a moderator of the association between perceived stress and smartphone use.

H10a-c: Social support moderates the associations between perceived stress and the three types of smartphone use (mass media, person-to-person, and social networking). The association between perceived stress and the three types of smartphone use will be stronger when people have low levels of social support comparted to when they have high levels of social support. 


\section{Conceptual Model}

Overall, this study aims to examine the reinforcement effect of PSU on perceived stress and later depression and the buffering effect of social support on the associations between perceived stress, smartphone use, and PSU, as well as explore the potential effects of differential types of smartphone use. Smartphone ownership among American young adults has nearly reached the saturation point (Taylor \& Silver, 2019) with PSU a growing concern. Although PSU has been found to be associated with multiple psychosocial constructs (Demirci et al., 2015; Elhai, Dvorak, et al., 2017; Samaha \& Hawi, 2016), the directionality and underlying mechanisms of these associations are underdetermined, and the theorization is unclear. Under this condition, the current study answers a series of important questions regarding how, when, and why smartphones are related to psychological well-beingdepression in this case. Together, this study uses a two-wave examination to test a reinforcement model of PSU, which theorizes the cause and consequence of PSU and the corresponding underlying mechanisms and boundary conditions.

The conceptual model of this study is shown in Figure 1.

Figure 1. Conceptual Model.

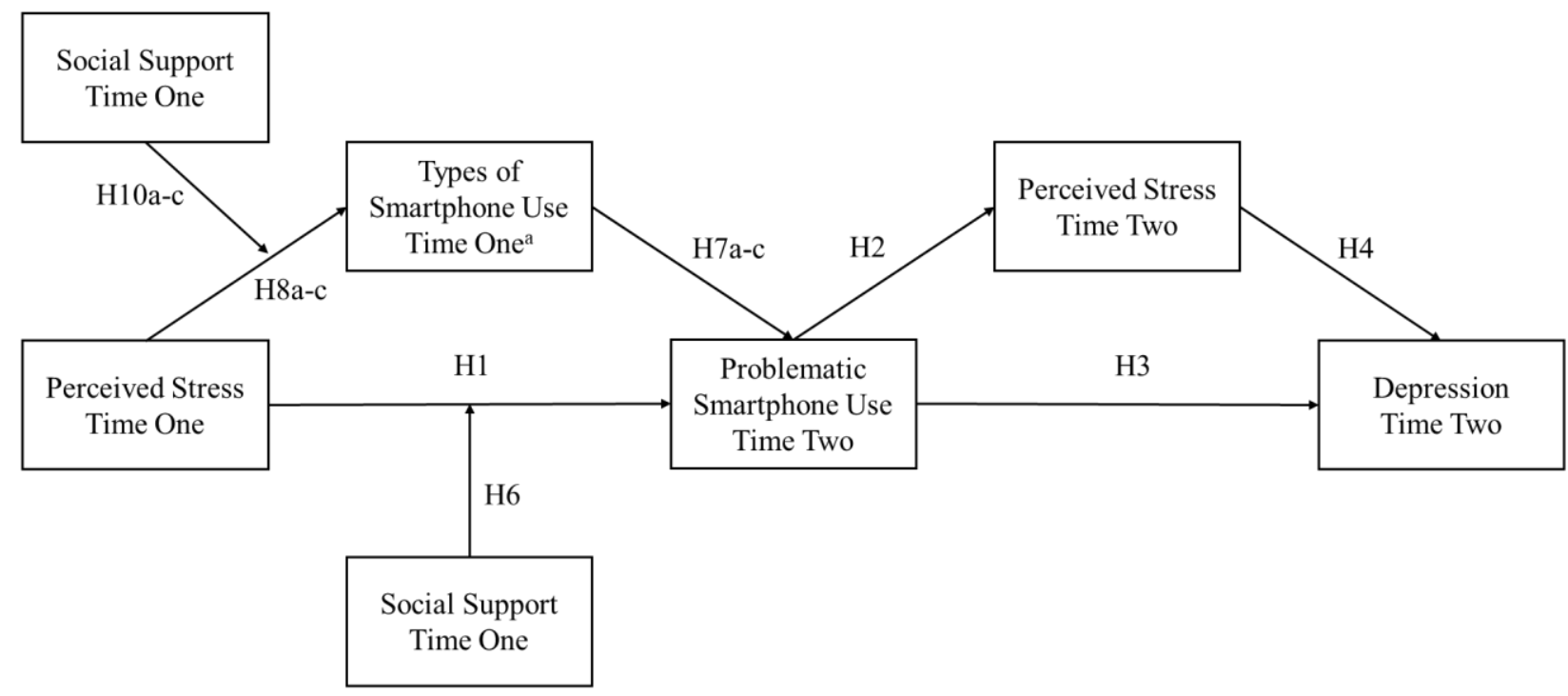

Note. ${ }^{\text {a }}$ For the purpose of clarity the three types of smartphone use have been combined in the figure (person-to-person smartphone use, social networking smartphone use, and mass media smartphone use).

\section{Methods}

\section{Recruitment}

After receiving approval from the authors' Institutional Review Board, we recruited college students enrolled in either communication or sociology courses from three large public universities across the United States. The schools were located in the American Southwest, the Midwest, and Southeast. Participants were asked to complete a two-wave online survey via Qualtrics. The first wave of data collection occurred at the beginning of 2018 (January and February) and the second wave of data collection took place during April 2018. Participants were asked to create a unique identifier in order for the researchers to link participation across waves.

\section{Sample}

A total of 816 participants were originally contacted to take part in the study. In the first wave of data collection, 585 participants completed the data collection (71.7\%). In the second wave 590 participants completed the data collection (72.3\%). That noted, not everyone who participated in the first wave of data collection took part in the 
second wave and there were individuals who took part at wave two who did not take part in wave one. As such, the total number of participants who completed both waves of data collection was 464 .

For the current study, we have only included respondents who were not in romantic relationships at the time of the data collections; this left us with 222 participants. We only included respondents who were single because this study was paired with another study that investigated smartphone use in romantic relationships, and thus those who were in romantic relationships were tasked with answering questions about their romantic relationships and smartphone use. Those who were not in romantic relationships were offered this alternative set of questions asking about their social support and perceived stress, which were the current study's focus. There was only one statistically significant difference between the partnered and unpartnered samples, as those in romantic relationships were older on average $\left(t(462)=-3.16, p=.002 ; M_{\text {partnered }}=20.03, M_{\text {unpartnered }}=19.60\right)$. To test whether there were differences between participants who completed both waves of data collection versus those who left after the first round, independent sample t-tests were conducted for all variables of interest. There were no significant differences between those that completed and those that did not, indicating that there were no attrition effects.

The mean age of participants in the sample at wave one was 19.60 years $(S D=1.30)$ and $60 \%$ were female $(n=$ 133). Participants in the study racially identified as white $(n=136,61.3 \%)$, African American $(n=10,4.5 \%)$, Asian/Pacific Islander ( $n=44,19.8 \%)$, Latino/a $(n=16,7.2 \%)$, Native American $(n=1,0.5 \%)$, "other" $(n=2,0.9 \%)$, and multi-racial ( $n=13,5.9 \%)$. Among the universities that participated, 50.9\% $(n=113)$ came from the university in the Midwest region, 33.8\% $(n=75)$ from the southwestern university, and 15.3\% $(n=34)$ from the southeastern university.

\section{Measures}

\section{Perceived Stress}

To measure perceived stress among participants, the researchers used the Perceived Stress Scale (Cohen et al., 1983). This is a 10-item scale that asks participants how often they have felt certain indicators of stress in the last month. For example, participants were asked "in the last month, how often have you felt that you are unable to control the important things in your life?" and "in the last month, how often have you been able to control irritations in your life?" (reverse scored). All questions were answered on a 5 -point scale ( $0=$ never to $4=$ very often) and were averaged to create the measure of perceived stress (Wave one: $\alpha=.85$; Wave two: $\alpha=.86$; see Table 1 for all means, standard deviations and zero-order correlations for variables of interest). Higher scores indicated greater perceived stress.

\section{Social Support}

The Interpersonal Support Evaluation List (Cohen et al., 1985) was used as this study's measure of social support. Participants were asked how true 12 statements were for them. Examples of the types of statements included "when I need suggestions on how to deal with a personal problem, I know someone I can turn to" and "if I were sick, I could easily find someone to help me with my daily chores/tasks". All statements were measured on a 4point scale $(0=$ definitely false to $3=$ definitely true $)$ and averaged, with higher scores indicating more access to interpersonal support (Wave one: $a=.89$ ).

\section{Smartphone Use}

To assess smartphone use, we asked participants to estimate their smartphone use on a typical day. They were asked to report how many minutes each day they used their smartphones in eight specific ways: talking on the phone, texting, using email, surfing the internet, using social networking applications (e.g., Snapchat, Twitter), using FaceTime and other video chat applications, playing videogames, and using news applications. Use was then split into three domains, there was person-to-person smartphone use (e.g., phoning, texting, email, and video chatting), mass media use (e.g., surfing the internet, playing videogames, and using news apps), and social networking use. Because of significant positive skew for all three measures, the three types of use were transformed using a log transformation. 
Kim and colleagues' (D. Kim et al., 2014) measure of smartphone addiction proneness was used for the current study; however, as others have suggested, such measures likely tap into problematic use rather than addiction (Griffiths, 2019). This is a 15-item scale with participants asked about their level of agreement regarding their smartphone use (e.g., "I panic when I cannot use my smartphone"). All questions employed a 4-point scale $(0=$ strongly disagree to 3 = strongly agree; Wave two: $\mathrm{a}=.89$ ).

\section{Depression}

The measure of depression used for this study was the 10-item Center for Epidemiologic Studies Depression Scale (Radloff, 1977). The scale asks participants to indicate how many days in the last week they felt a particular way. For example, participants were asked if they were "bothered by things that usually don't bother me" or "I felt fearful". Questions were answered using a four-point scale $(0=$ rarely or none of the time (less than one day) to $3=$ most to all of the time (5-7 days)) and were averaged to create the measure (Wave two: $a=.83$ ).

Table 1. Means, Standard Deviations, and Zero-Order Correlations for All Variables of Interest.

\begin{tabular}{|c|c|c|c|c|c|c|c|c|c|c|}
\hline Variable & $M(S D)$ & 2. & 3. & 4. & 5. & 6. & 7. & 8. & 9. & 10. \\
\hline 1. Participant gender $($ women $=1)$ & $60 \%$ & $-.239^{* * *}$ & .103 & $.156^{*}$ & $.265^{* \star *}$ & $-.153^{*}$ & $.300^{* * *}$ & .108 & $.185^{* *}$ & .008 \\
\hline 2. Participant age & $19.60(1.303)$ & & $-.170^{*}$ & .034 & -.001 & -.102 & $-.233^{* * *}$ & -.046 & $-.130^{+}$ & -.062 \\
\hline 3. W1- Perceived stress & $1.856(0.544)$ & & & $-.509^{* \star *}$ & .045 & $.119^{+}$ & $.173^{* * *}$ & $.665^{* \star *}$ & $.382^{* * *}$ & $.594^{\star \star *}$ \\
\hline 4: W1- Social support & $2.194(0.579)$ & & & & $.226^{* *}$ & $-.139^{*}$ & $.148^{*}$ & $-.417^{\star \star \star}$ & $-.273^{* \star \star}$ & $-.534^{\star * \star}$ \\
\hline 5. W1-SU-Person-to-person & $2.077(0.376)$ & & & & & $.223^{* *}$ & $.531^{* * *}$ & -.007 & .056 & -.014 \\
\hline 6. W1-SU- Mass mediated & $1.902(0.425)$ & & & & & & $.201^{* *}$ & .084 & .055 & $.250^{* \star *}$ \\
\hline 7. W1-SU-Social networking & $1.873(0.522)$ & & & & & & & $.121^{+}$ & $.296^{* * *}$ & .077 \\
\hline 8. W2- Perceived stress & $1.89(0.560)$ & & & & & & & & $.542^{* * *}$ & $.756^{* \star *}$ \\
\hline 9. W2- PSU & $1.226(0.478)$ & & & & & & & & & $.436^{* \star *}$ \\
\hline 10. W2- Depression & $0.993(0.574)$ & & & & & & & & & \\
\hline
\end{tabular}

Note. $\mathrm{SU}=$ smartphone use, $\mathrm{W} 1$ = wave one, $\mathrm{W} 2$ = wave two, PSU = problematic smartphone use.

${ }^{+} p<.10,{ }^{*} p<.05,{ }^{*} p<.01,{ }^{* *} p<.001$.

\section{Analysis Strategy}

All hypotheses were tested using Preacher and Hayes' mediation and moderation macro for SPSS using 5,000 bootstrapped samples (Hayes, 2017). Given that we only had two waves of data, we are unable to fully test the conceptual model using one comprehensive statistical model. Therefore, we decided to use two separate statistical models to test the proposed conceptual model. For the model with PSU as the dependent variable, model 8 was used with each type of smartphone use entered in parallel as mediators. The moderator for this model was social support and the independent variable was perceived stress at wave one, both of these variables were centered prior to inclusion in the models. Two covariates were included in this model, participant gender and participant age.

The model with depression as the dependent variable was tested with PROCESS model 4. Perceived stress at wave two was the mediator and PSU was the primary independent variable. We entered the variables from the first model as covariates to determine the unique contribution of both PSU and perceived stress on depression. The significance threshold was set at $p<.05$ for all tests.

\section{Results}

See Table 2 for a record of all hypotheses and whether they were supported. The first model featured social support as a moderator with the various aspects of smartphone use included as parallel mediators (see Table 3 ). The first of the mediators tested was person-to-person smartphone use and the model was significant $(R=0.36$, $\left.R^{2}=0.13, F(5,215)=6.42, p<0.001\right)$. Of the variables included, increased stress significantly predicted increased 
person-to-person smartphone use, increased social support was associated with more use, and women used their phones in this manner more. The interaction of perceived stress and social support was not significant. The second mediator tested was mass mediated smartphone use and the model was significant $\left(R=.24, R^{2}=.06, F(5,215)=\right.$ $2.72, p=.021$ ). The only variable that significantly predicted this type of smartphone use was gender as men were more likely to use their smartphones in this way.

Table 2. Summary of Hypotheses Testing Results.

\begin{tabular}{|c|c|c|}
\hline \multicolumn{2}{|c|}{ Hypotheses } & \multirow{2}{*}{$\begin{array}{c}\text { Results } \\
\text { Supported }\end{array}$} \\
\hline $\mathrm{H} 1$ & Perceived stress is positively associated with later PSU. & \\
\hline $\mathrm{H} 2$ & PSU is positively associated with perceived stress. & Supported \\
\hline H3 & PSU is positively associated with depression. & Rejected \\
\hline $\mathrm{H} 4$ & Perceived stress is positively associated with depression. & Supported \\
\hline H5 & Perceived stress mediates the association between PSU and depression. & Supported \\
\hline H6 & Social support moderates the association between perceived stress and PSU. & Rejected \\
\hline \multirow[t]{3}{*}{$\mathrm{H} 7$} & a: Mass media smartphone use is positively associated with later PSU. & Rejected \\
\hline & b: Person-to-person smartphone use is positively associated with later PSU. & Rejected \\
\hline & c: Social networking smartphone use is positively associated with later PSU. & Supported \\
\hline \multirow[t]{3}{*}{$\mathrm{H} 8$} & a: Perceived stress is positively associated with mass media smartphone use. & Rejected \\
\hline & b: Perceived stress is positively associated with person-to-person smartphone use. & Supported \\
\hline & c: Perceived stress is positively associated with social networking smartphone use. & Supported \\
\hline \multirow[t]{3}{*}{ H9 } & a: Mass media smartphone use mediates the association between perceived stress and PSU. & Rejected \\
\hline & b: Person-to-person smartphone use mediates the association between perceived stress and PSU. & Rejected \\
\hline & c: Social networking smartphone use mediates the association between perceived stress and PSU. & Supported \\
\hline \multirow[t]{3}{*}{$\mathrm{H} 10$} & a: Social support moderates the associations between perceived stress and mass media smartphone use. & Rejected \\
\hline & b: Social support moderates the associations between perceived stress and person-to-person smartphone use. & Rejected \\
\hline & c: Social support moderates the associations between perceived stress and social networking smartphone use. & Supported \\
\hline
\end{tabular}

Table 3. Moderated-Mediation Regression Models Predicting the Three Smartphone Use Mediators and Problematic Smartphone Use.

\begin{tabular}{|c|c|c|c|c|c|c|c|c|}
\hline \multirow{2}{*}{ Variable } & \multicolumn{2}{|c|}{$\begin{array}{l}\text { W1 Person-to-person } \\
\text { Smartphone Use }\end{array}$} & \multicolumn{2}{|c|}{$\begin{array}{l}\text { W1 Social Networking } \\
\text { Smartphone Use }\end{array}$} & \multicolumn{2}{|c|}{$\begin{array}{l}\text { W1 Mass Media } \\
\text { Smartphone Use }\end{array}$} & \multicolumn{2}{|c|}{$\begin{array}{l}\text { W2 Smartphone } \\
\text { Dependency }\end{array}$} \\
\hline & $b$ & $S E$ & $b$ & $S E$ & $b$ & $S E$ & $b$ & $S E$ \\
\hline Constant & $1.560^{\star \star \star}$ & 0.384 & $2.868^{* * *}$ & 0.513 & $2.803^{* * *}$ & 0.451 & 0.814 & 0.513 \\
\hline Participant age & 0.021 & 0.019 & $-0.059^{*}$ & 0.026 & $-0.041^{+}$ & 0.022 & 0.006 & 0.024 \\
\hline $\begin{array}{l}\text { Participant gender } \\
(1=\text { female) }\end{array}$ & $0.170^{* *}$ & 0.052 & $0.217^{* *}$ & 0.069 & $-0.158^{*}$ & 0.061 & $0.128^{+}$ & 0.066 \\
\hline W1 Perceived stress & $0.121^{*}$ & 0.053 & $0.239^{* *}$ & 0.070 & 0.065 & 0.062 & $0.191^{* *}$ & 0.064 \\
\hline W1 Social support & $0.185^{* * *}$ & 0.051 & $0.259^{* * *}$ & 0.068 & 0.040 & 0.060 & $-0.164^{*}$ & 0.064 \\
\hline $\begin{array}{l}\text { W1 Social support X W1 } \\
\text { Perceived stress }\end{array}$ & -0.027 & 0.072 & $-0.198^{*}$ & 0.096 & -0.029 & 0.085 & -0.042 & 0.087 \\
\hline W1 Person-to-person SU & & & & & & & -0.128 & 0.095 \\
\hline W1 Social networking SU & & & & & & & $0.280^{* \star \star}$ & 0.070 \\
\hline W1 Mass media SU & & & & & & & -0.021 & 0.073 \\
\hline
\end{tabular}

Note. SU = smartphone use.

${ }^{+} p<.10,{ }^{*} p<.05,{ }^{* *} p<.01,{ }^{* * *} p<.001$.

The final mediator tested was social networking smartphone use and the model was significant $\left(R=.44, R^{2}=.19\right.$, $F(5,215)=10.24, p<.001)$. All variables in this model significantly predicted social networking smartphone use: perceived stress was associated with more use, social support was associated with more use, women used more social networking apps on their smartphones, and older participants spent less time using their smartphones for social networking. Of particular note, we found a significant moderating effect for social support. As seen in Figure 2 , the link between perceived stress and social networking smartphone use was strongest for those people who 
reported not having much social support while those individuals who reported having the most social support were not significantly different on perceived stress and social networking smartphone use.

Figure 2. Plotted Interaction Effect for Social Support and Perceived Stress on Social Networking Smartphone Use.

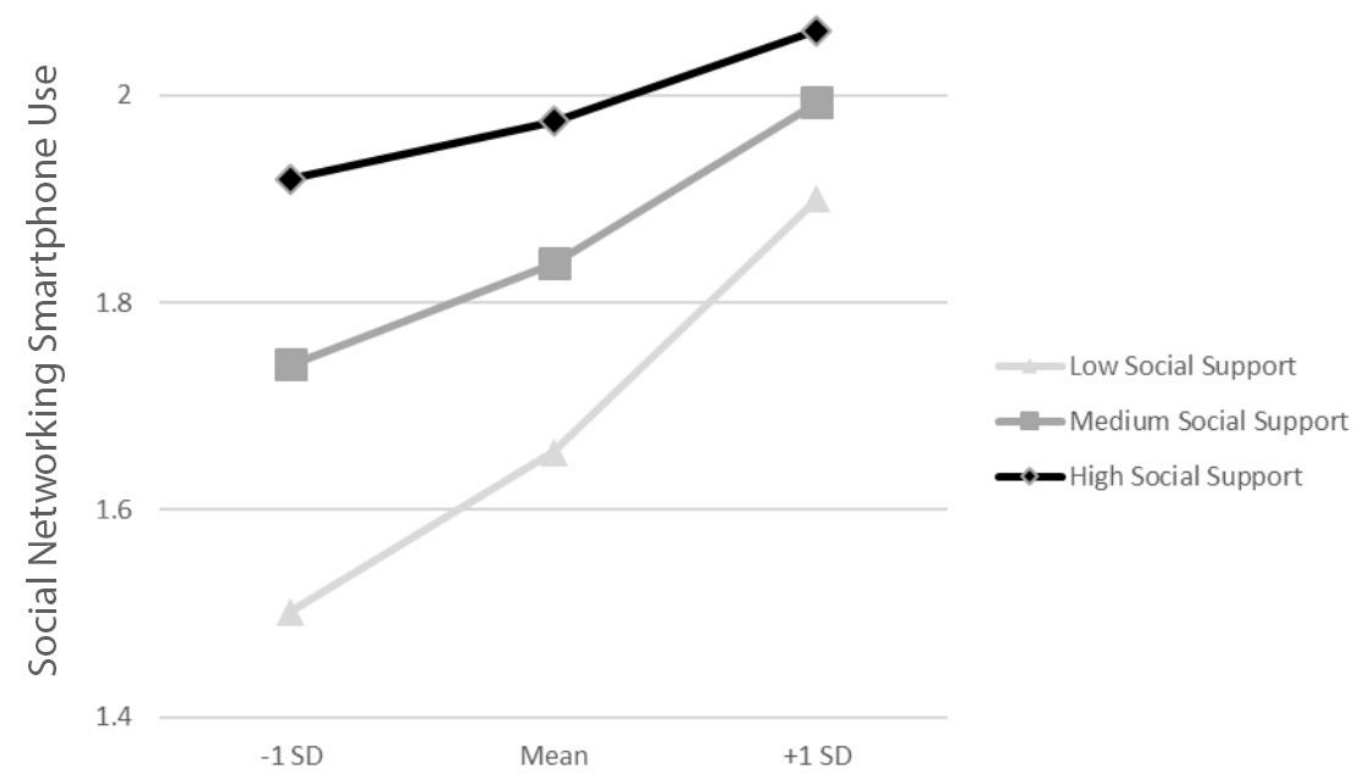

Perceived Stress

The model predicting PSU at time two was significant $\left(R=.50, R^{2}=.25, F(8,212)=8.72, p<.001\right)$. The results showed that increased perceived stress was associated with more PSU, increased social networking smartphone use was associated with increased PSU, and less social support was linked to more PSU. The interaction term of perceived stress and social support was not significant.

As Table 4 shows, the indirect effects of perceived stress on PSU via the three types of smartphone use yielded interesting results. For person-to-person and mass mediated smartphone use, there were no significant indirect effects. However, for social networking smartphone use, there were significant indirect effects but just for those individuals who reported having low $(b=0.10,95 \% \mathrm{Cl}[0.03,0.21])$ and medium $(b=0.06,95 \% \mathrm{Cl}[0.02,0.14])$ levels of social support.

Table 4. Conditional Indirect Effects for Perceived Stress on Problematic Smartphone Use With Social Support as Moderator.

\begin{tabular}{llcccc}
\hline Mediator & Moderator Level & Effect & SE & LLCI & ULCI \\
\hline Person-to-person smartphone use & Low & -0.018 & 0.021 & -0.066 & 0.016 \\
& Medium & -0.015 & 0.018 & -0.058 & 0.012 \\
& High & -0.014 & 0.019 & -0.061 & 0.012 \\
\hline Social networking smartphone use & Low & 0.101 & 0.047 & 0.028 & 0.212 \\
& Medium & 0.064 & 0.031 & 0.017 & 0.135 \\
& High & 0.036 & 0.026 & -0.004 & 0.097 \\
\hline Mass media smartphone use & Low & -0.002 & 0.009 & -0.021 & 0.015 \\
& Medium & -0.001 & 0.007 & -0.017 & 0.012 \\
& High & -0.001 & 0.007 & -0.018 & 0.013 \\
\hline
\end{tabular}

Note. Lines that are italicized are significant.

The second step in the total model tested the mediational influence of perceived stress at time two on PSU and depression (see Table 5). For this set of tests, each of the variables that were included in the first set of analyses were included as covariates. The regression model predicting perceived stress at time two was significant $(R=.75$, $\left.R^{2}=.56, F(9,211)=30.33, p<.001\right)$. Of particular note, we found that PSU was positively associated with perceived 
stress. We also found that perceived stress at time one significantly predicted stress at time two and that the previously entered interaction term of perceived stress and social support at time one was significant.

Table 5. Regression Models for Mediational Testing of Perceived Stress on Depression.

\begin{tabular}{|c|c|c|c|c|c|c|}
\hline \multirow{2}{*}{ Variable } & \multicolumn{2}{|c|}{ W2 Perceived Stress } & \multicolumn{2}{|c|}{$\begin{array}{l}\text { W2 Depression with } \\
\text { W2 Perceived Stress }\end{array}$} & \multicolumn{2}{|c|}{$\begin{array}{l}\text { W2 Depression without } \\
\text { W2 Perceived Stress }\end{array}$} \\
\hline & $b$ & $S E$ & $b$ & $S E$ & $b$ & $S E$ \\
\hline Constant & 0.695 & 0.462 & -0.589 & 0.423 & -0.154 & 0.510 \\
\hline Participant age & $0.041^{+}$ & 0.021 & -0.002 & 0.019 & 0.023 & 0.023 \\
\hline Participant gender (1 = female) & 0.045 & 0.060 & -0.005 & 0.054 & 0.023 & 0.066 \\
\hline W2 Perceived stress & & & $0.627^{\star \star \star}$ & 0.063 & & \\
\hline W2 PSU & $0.410^{* * *}$ & 0.061 & 0.022 & 0.062 & $0.280^{* * *}$ & 0.068 \\
\hline W1 Perceived stress & $0.541^{* * *}$ & 0.059 & 0.050 & 0.063 & $0.389^{\star \star \star}$ & 0.065 \\
\hline W1 Social support & -0.074 & 0.058 & $-0.212^{\star \star \star}$ & 0.053 & $-0.259^{\star \star \star}$ & 0.064 \\
\hline W1 Social support X W1 Perceived stress & $0.168^{*}$ & 0.072 & -0.074 & 0.071 & 0.031 & 0.364 \\
\hline W1 Person-to-person SU & -0.058 & 0.086 & 0.024 & 0.078 & -0.012 & 0.095 \\
\hline W1 Social networking SU & -0.030 & 0.065 & -0.026 & 0.059 & -0.045 & 0.072 \\
\hline W1 Mass media SU & 0.038 & 0.066 & $0.215^{* * *}$ & 0.060 & $0.238^{* *}$ & 0.072 \\
\hline
\end{tabular}

Note. SU = smartphone use; PSU = problematic smartphone use.

${ }^{+} p<.10,{ }^{*} p<.05,{ }^{* *} p<.01,{ }^{* * *} p<.001$.

The model predicting depression at time two was significant $\left(R=.81, R^{2}=.66, F(10,210)=40.38, p<.001\right)$. The results revealed that perceived stress at time two was associated with depression as increased stress meant increased depression and that PSU was not associated with depression. We further found that mass mediated smartphone use was associated with more depression and time one interpersonal support was linked to less depression.

The model predicting depression without perceived stress included was significant $\left(R=.70, R^{2}=.50, F(9,211)=\right.$ $22.95, p<.001)$. What this model showed was that time two PSU did significantly predict depression. The results also indicated that mass mediated smartphone use was associated with increased depression, perceived stress at time one was linked to more depression, and more interpersonal support at time one was associated with less depression. The results further showed that perceived stress fully mediated the link between PSU and depression as the direct effect was not significant $(b=0.02,95 \% \mathrm{Cl}[-0.10,0.14], t=0.37, p=.715)$ but the indirect effect was $(b=0.26,95 \% \mathrm{Cl}[0.15,0.37])$.

\section{Discussion}

The current study had three primary aims. First, this study examined the reinforcement effect of PSU on perceived stress and subsequent depression. Second, the study explored the moderating effect of social support on the association between perceived stress and PSU. Lastly, this study investigated the associations among different type of smartphone use, perceived stress, social support, and PSU. Our results showed that there was a reinforcement effect for PSU on stress, which was associated with an increase in depressive symptoms. In addition, contrary to our expectations, we only found that social support moderated the association between stress and PSU for social networking smartphone use. Furthermore, we found that perceived stress significantly predicted person-to-person and social networking smartphone use (see Table 2 for a full accounting of which hypotheses were supported). Together, by conducting a two-wave examination, this study tested a reinforcement model of PSU, which theorizes the cause and consequence of PSU and the corresponding underlying mechanisms and boundary conditions.

\section{The Reinforcement Effects of PSU on Perceived Stress}

First, the results showed that there was a bidirectional relationship between perceived stress and PSU (H1 and H2). Namely, perceived stress predicted later PSU, and PSU also predicted later perceived stress. The result that 
perceived stress was predictive of later PSU is consistent with the stress-coping framework and findings of previous literature on other addictive behaviors. Namely, stressed people may develop PSU as a type of maladaptive coping strategy to cope with perceived stress. Also, as other studies suggested, stressful life events or environments may change individuals' stress responsiveness, which can increase the likelihood of the onset of addictive disorders (Del Giudice et al., 2011; Kreek et al., 2005). In addition, this finding is also in line with the assumptions of CIUT, which proposes that stressors motivate people to excessively use internet technology for alleviating negative emotions and stress (Kardefelt-Winther, 2014a). Therefore, the finding that perceived stress was associated with later PSU supports the contention that PSU may function similarly as other addictions by sharing the same risk factor-stress, and the function of problematic use on smartphones is to cope with perceived stress.

The results also extend the literature by finding that PSU predicted later perceived stress. Particularly, PSU may reinforce perceived stress for those with high levels of stress. While people use smartphones to alleviate perceived stress, our findings showed that increased psychological reliance on smartphones is not a favorable coping approach to deal with this stress. The social and vocational impairments caused by PSU (Tokunaga, 2014) may become new or reinforced stressors further reducing people's perceived control over their lives, and thus strengthen their perceived stress. Future research should explore the underlying mechanisms between PSU and later perceived stress such as reduced social support or decreased vocational effectiveness. Given the reinforcement effects, the relationship between perceived stress and PSU might become a downward spiral. In other words, through PSU, perceived stress might be reinforced especially for people with high levels of stress, and reinforced perceived stress breeds increased PSU over time.

\section{The Mediating Effect of Perceived Stress}

Although previous research showed that PSU is consistently linked to depression (Aker et al., 2017), the question regarding how the effect happens is underexplored. Our results extend the previous research by showing that perceived stress fully mediated the association between PSU and depression (H5). Namely, PSU was associated with increased perceived stress, which, in turn, was positively associated with depression. The mediating effect of perceived stress is not surprising as stress is consistently associated with depression (Van Praag, 2004). However, the full mediation model of perceived stress contributes to the existing literature by suggesting that exploring the role of perceived stress is likely key to understanding the deleterious effect of PSU on depression. In order to further unpack the association between PSU and depression, future research should explore other possible mediators such as anxiety and social support.

\section{Differential Effects of Smartphone Use}

Our results revealed that perceived stress was associated with both person-to-person and social networking smartphone use ( $\mathrm{H} 8 \mathrm{~b}$ and $\mathrm{H} 8 \mathrm{c}$ ). These findings are also consistent with the assumptions of CIUT as people may use online communication and SNSs to alleviate their perceived stress. Specifically, people with high perceived stress may use person-to-person communication and SNSs for mood management and social support seeking. There are several hints in the literature toward this explanation (Johnson \& Knobloch-Westerwick, 2014; Leung, 2007). For example, Leung (2007) found that stressful life events are associated with internet use for mood management and social compensation motives as internet consumption for these two motives increases adolescents' perceived social support. In addition, Johnson and Knobloch-Westerwick (2014) found that exposure to downward social comparison information on SNSs can reduce negative emotions. Although the association between perceived stress and smartphone use may work in the opposite direction (i.e., person-to-person and social networking smartphone use may cause increased perceived stress), a number of studies have suggested that smartphone use for multimodal communication is beneficial for people's well-being instead of causing negative outcomes (Bae, 2019; Cho, 2015).

Regarding the association between perceived stress and smartphone use, inconsistent with our hypothesis, this study did not find an association between perceived stress and mass mediated smartphone use (H8a). It might be because college students primarily experience interpersonal and academic stress in their daily lives (Aselton, 2012), and they thus prefer to use smartphones for communicative purposes to seek social support from others in order to reduce their stress. Consequently, because mass mediated smartphone use is not useful for providing 
social support that functions as a reducer of perceived stress, we did not find the association between perceived stress and mass media smartphone use. Supporting this explanation, our results also showed that only personto-person $(b=0.185, p<.001)$ and social networking smartphone use $(b=0.259, p<.001)$ were positively associated with social support, but mass media smartphone use was not significantly associated with social support (see Table 3). Future research should investigate the associations between different types of stressors and different types of smartphone use.

Our results also showed that social networking smartphone use was positively associated with later PSU (H7c). This finding is in line with the results of previous research, which found that socially-oriented smartphone use was positively associated with PSU (Elhai, Levine, et al., 2017). It is possible that SNS users may develop a habit of mindlessly or actively checking their SNS notifications and others' updates, and this mindless or habitual smartphone use may cause PSU over time (Van Deursen et al., 2015). Future research should further explore the role of habitual smartphone use. In addition, this study did not find a relationship between person-to-person smartphone use and PSU (H7b). Although previous research found that socially-oriented smartphone use can be related to PSU (Elhai, Levine, et al., 2017), these studies did not distinguish person-to-person and social networking use. Our results suggest that the benefits of person-to-person smartphone use such as increased social support (Cho, 2015) may prevent the onset of PSU. Therefore, future research should split smartphone use into personto-person and social networking use to determine whether these findings hold.

Previous literature suggests that process-oriented smartphone use or smartphone use for information seeking, entertainment seeking, and gaming was directly related to PSU (Bae, 2017; Van Deursen et al., 2015); however, this study found that mass media smartphone use did not significantly predict later PSU (H7a). It might be because of motivations of smartphone use. According to CIUT, the interaction between stress and escapism causes digital addiction (Kardefelt-Winther, 2014b). However, in the current study, perceived stress was not a cause of mass mediated smartphone use. Therefore, without the motivations of alleviating stress or escaping from stressors, mass mediated smartphone use might not lead to PSU. Future research should take into account the motivations of mass mediated use when exploring the association between such use and PSU.

\section{The Moderating Effect of Social Support}

Our results further showed that social support moderated the link between perceived stress and social networking smartphone use ( $\mathrm{H} 10 \mathrm{c})$. Namely, only for people with low to medium levels of social support, perceived stress was associated with increased social networking smartphone use. This finding supports the assumptions of stressbuffering model of social support (Cohen \& Wills, 1985). For those with high levels of social support, their perceived stress can be alleviated by using available supportive resources. However, for those with fewer supportive resources, they need to use SNSs on their smartphones to either seek social support or to assist in managing their moods. In addition, our results also showed that for those who had low or medium levels of social support, perceived stress indirectly predicted PSU via social networking smartphone use (H9c). This finding offers support for the idea that the interaction between social support and perceived stress can lead to social networking smartphone use, which, in turn, predicts later PSU. However, our results did not show that social support moderated the association between perceived stress and PSU (H6). It might be because the connection between the types of social support and the types of stressors matters (Cutrona, 1990). Specifically, the optimal matching model of stress and social support assumes that the most beneficial outcomes occur when the types of social support match with the types of stressful life events that support-seekers experience (Cutrona, 1990; Cutrona \& Russell, 1990). Consequently, the lack of an interaction effect on PSU may be because of the mismatch of the types of social support and stressors. Therefore, future studies should explore the buffering effects related to specific types of social support on the corresponding types of stressful events.

\section{Implications for Research and Practice}

This study found the reinforcement effects of PSU on perceived stress and the mediating effect of perceived stress on the association between PSU and depression. These findings contribute to our theoretical understandings of the risk factors and consequences of PSU. Especially, the bidirectional relationships between perceived stress and depression indicate the existence of a downward spiral or vicious circle. Also, the findings related to smartphone use can help researchers distinguish different roles that different types of smartphone use play. 
In addition, it is necessary to acknowledge that given the rates of smartphone ownership/use among young people, smartphones play an important role in young people's mental health maintenance. Therefore, understanding the directionality of the relationship between smartphone engagement and depression and the effects of perceived stress and social support on smartphone engagement can shed light on how to maintain young people's mental health. Health practitioners should consult with young people about their smartphone use and help them understand the links between perceived stress, smartphone engagement, and depression. Especially, although young people may intend to use smartphones to reduce their stress, our results have shown problematic smartphone use can result in increased perceived stress and later depression. Therefore, in order to protect their well-being, young people should be suggested to use healthy means to cope with stress.

\section{Limitations}

There are some limitations with the current study that ought to be addressed. First, this study used a convenience sample comprised entirely of college students. To be clear, this is an important population to study particularly since they are the most consistent users of smartphones (Taylor \& Silver, 2019). However, in order to confirm whether the results can be generalized to the population of young adults, future research should investigate these associations using more diverse samples. Second, the current study only included single people because those who were in romantic relationships were tasked with answering questions about their romantic relationships and smartphone use. Future research may test the validity of these results with people with romantic relationships. Third, this study used two waves of data to test the reinforcement model of PSU and the role of different types of smartphone use. Although this study offered preliminary results regarding the ordering of these constructs, in order to fully investigate the mediation model, future research should collect multiple waves of data from participants.

Fourth, although this study offered evidence for potential causal relationships, experimental studies are still required to examine the causal relationships among these constructs. In addition, other variables, such as selfregulation, impulsivity, functional and vocational impairment, may also need to be involved in the relationships this study examined. Therefore, future studies should investigate the effects of these variables on smartphone use and PSU as well. The last limitation associated with the current study is that our measure of smartphone use relied on self-report from participants. In general, measuring media use is a challenge (see de Vreese \& Neijens, 2016) and smartphone use is certainly not immune from these measurement concerns, particularly self-report data (Ellis, 2019). However, because of the challenges associated with collecting data from multiple locations in the United States and across two waves, this method seemed the most appropriate. Moreover, research on selfreported smartphone/mobile phone use does indicate that it is appropriately associated with direct observations of smartphone use (see Boase \& Ling, 2013; Lee et al., 2017). That noted, future studies should use direct observations of smartphone use (e.g., using applications like Moment or Screen Time for the iPhone) to validate the current findings.

\section{Conclusion}

Smartphones provide users with an array of tools to stay connected with the world and, over the last decade, have become essential devices. They help us to connect with friends and loved ones in a multitude of ways, they help us to manage our day-to-day affairs, and they even offer a needed distraction when we need to kill some time (e.g., playing a game or scrolling through Twitter while waiting for our car to be repaired). Yet, there is accumulating evidence that for those people who get overly attached to their devices, they are likely to experience a range of negative outcomes like loneliness and depressive symptoms. The current study explored the reinforcement effects of PSU on stress and depression. As well as the potential buffering effects of social support on this relationship and whether different types of smartphone use may be implicated. Our results revealed that PSU and perceived stress potentially reinforce one another, and that stress fully mediates the relationship between PSU and depression among college students. The results also suggest that individuals who are faced with increased stress are more likely to use their smartphones to connect to others around them either with person-to-person use or social networking use. Yet, increased social networking use possibly exacerbates the effect of stress on PSU for those individuals with less social support. These findings point to important avenues of inquiry regarding how our use and engagement with smartphones affect our well-being and also how we use these devices to obtain social support. 


\section{References}

Aker, S., Şahin, M. K., Sezgin, S., \& Oğuz, G. (2017). Psychosocial factors affecting smartphone addiction in university students. Journal of Addictions Nursing, 28(4), 215-219. https://doi.org/10.1097/JAN.0000000000000197

Alhassan, A. A., Alqadhib, E. M., Taha, N. W., Alahmari, R. A., Salam, M., \& Almutairi, A. F. (2018). The relationship between addiction to smartphone usage and depression among adults: A cross sectional study. BMC Psychiatry, 18(1), Article 148. https://doi.org/10.1186/s12888-018-1745-4

American Psychiatric Association. (2013). Diagnostic and statistical manual of mental disorders (5th ed.). https://doi.org/10.1176/appi.books.9780890425596

Aselton, P. (2012). Sources of stress and coping in American college students who have been diagnosed with depression. Journal of Child and Adolescent Psychiatric Nursing, 25(3), 119-123. https://doi.org/10.1111/j.17446171.2012.00341.x

Bae, S.-M. (2017). The relationship between the type of smartphone use and smartphone dependence of Korean adolescents: National survey study. Children and Youth Services Review, 81, 207-211. https://doi.org/10.1016/j.childyouth.2017.08.012

Bae, S.-M. (2019). The relationship between smartphone use for communication, social capital, and subjective well-being in Korean adolescents: Verification using multiple latent growth modeling. Children and Youth Services Review, 96, 93-99. https://doi.org/10.1016/j.childyouth.2018.11.032

Beranuy, M., Carbonell, X., \& Griffiths, M. D. (2013). A qualitative analysis of online gaming addicts in treatment. International Journal of Mental Health and Addiction, 11(2), 149-161. https://doi.org/10.1007/s11469-012-9405-2

Billieux, J. (2012). Problematic use of the mobile phone: A literature review and a pathways model. Current Psychiatry Reviews, 8(4), 299-307. https://doi.org/10.2174/157340012803520522

Boase, J., \& Ling, R. (2013). Measuring mobile phone use: Self-report versus log data. Journal of ComputerMediated Communication, 18(4), 508-519. https://doi.org/10.1111/jcc4.12021

Brand, M., Young, K. S., Laier, C., Wölfling, K., \& Potenza, M. N. (2016). Integrating psychological and neurobiological considerations regarding the development and maintenance of specific Internet-use disorders: An Interaction of Person-Affect-Cognition-Execution (I-PACE) model. Neuroscience \& Biobehavioral Reviews, 71, 252-266. https://doi.org/10.1016/j.neubiorev.2016.08.033

Carroll, L. (2013). Problem-focused coping. In M. D. Gellman \& J. R. Turner (Eds.), Encyclopedia of behavioral medicine (pp. 1540-1541). Springer. https://doi.org/10.1007/978-1-4419-1005-9_1171

Chapman, D. P., Perry, G. S., \& Strine, T. W. (2005). The vital link between chronic disease and depressive disorders. Preventing Chronic Disease, 2(1).www.cdc.gov/pcd/issues/2005/jan/04_0066.htm

Chiu, S.-I. (2014). The relationship between life stress and smartphone addiction on Taiwanese university student: A mediation model of learning self-efficacy and social self-efficacy. Computers in Human Behavior, 34, 49-57. https://doi.org/10.1016/j.chb.2014.01.024

Cho, J. (2015). Roles of smartphone app use in improving social capital and reducing social isolation. Cyberpsychology, Behavior, and Social Networking, 18(6), 350-355. https://doi.org/10.1089/cyber.2014.0657

Cohen, S., Kamarck, T., \& Mermelstein, R. (1983). A global measure of perceived stress. Journal of Health and Social Behavior, 24(4), 385-396. https://doi.org/10.2307/2136404 
Cohen, S., Mermelstein, R., Kamarck, T., \& Hoberman, H. M. (1985). Measuring the functional components of social support. In I. G. Sarason \& B. R. Sarason (Eds.), Social support: Theory, research and applications (pp. 73-94). Springer.

Cohen, S., \& Wills, T. A. (1985). Stress, social support, and the buffering hypothesis. Psychological Bulletin, 98(2), 310-357. https://doi.org/10.1037/0033-2909.98.2.310

Cutrona, C. E. (1990). Stress and social support-In search of optimal matching. Journal of Social and Clinical Psychology, 9(1), 3-14. https://doi.org/10.1521/jscp.1990.9.1.3

Cutrona, C. E., \& Russell, D. W. (1990). Type of social support and specific stress: Toward a theory of optimal matching. In B. R. Sarason, I. G. Sarason, \& G. R. Pierce (Eds.), Social support: An interactional view. (pp. 319-366). John Wiley \& Sons.

de Vreese, C. H., \& Neijens, P. (2016). Measuring media exposure in a changing communications environment. Communication Methods and Measures, 10(2-3), 69-80. https://doi.org/10.1080/19312458.2016.1150441

Del Giudice, M., Ellis, B. J., \& Shirtcliff, E. A. (2011). The adaptive calibration model of stress responsivity. Neuroscience and Biobehavioral Reviews, 35(7), 1562-1592. https://doi.org/10.1016/j.neubiorev.2010.11.007

Demirci, K., Akgönül, M., \& Akpinar, A. (2015). Relationship of smartphone use severity with sleep quality, depression, and anxiety in university students. Journal of Behavioral Addictions, 4(2), 85-92.

https://doi.org/10.1556/2006.4.2015.010

Dhillon, R., \& Arora, M. (2017). Perceived stress, self-efficacy, coping strategies and hardiness as predictors of depression. Journal of Psychosocial Research, 12(2), 325-333.

Ditzen, B., \& Heinrichs, M. (2014). Psychobiology of social support: The social dimension of stress buffering. Restorative Neurology and Neuroscience, 32(1), 149-162. https://doi.org/10.3233/RNN-139008

Dobkin, P. L., De Civita, M., Paraherakis, A., \& Gill, K. (2002). The role of functional social support in treatment retention and outcomes among outpatient adult substance abusers. Addiction, 97(3), 347-356.

https://doi.org/10.1046/j.1360-0443.2002.00083.x

Duke, É., \& Montag, C. (2017). Smartphone addiction, daily interruptions and self-reported productivity. Addictive Behaviors Reports, 6, 90-95. https://doi.org/10.1016/j.abrep.2017.07.002

Elhai, J. D., Dvorak, R. D., Levine, J. C., \& Hall, B. J. (2017). Problematic smartphone use: A conceptual overview and systematic review of relations with anxiety and depression psychopathology. Journal of Affective Disorders, 207, 251-259. https://doi.org/10.1016/j.jad.2016.08.030

Elhai, J. D., Levine, J. C., Dvorak, R. D., \& Hall, B. J. (2017). Non-social features of smartphone use are most related to depression, anxiety and problematic smartphone use. Computers in Human Behavior, 69, 75-82.

https://doi.org/10.1016/j.chb.2016.12.023

Ellis, D. A. (2019). Are smartphones really that bad? Improving the psychological measurement of technologyrelated behaviors. Computers in Human Behavior, 97, 60-66. https://doi.org/10.1016/j.chb.2019.03.006

Folkman, S., \& Lazarus, R. S. (1980). An analysis of coping in a middle-aged community sample. Journal of Health and Social Behavior, 21(3), 219-239. https://doi.org/10.2307/2136617

Fryer, S., Waller, G., \& Kroese, B. S. (1997). Stress, coping, and disturbed eating attitudes in teenage girls. International Journal of Eating Disorders, 22(4), 427-436. https://doi.org/10.1002/(SICI)1098-

108X(199712)22:4<427::AID-EAT8>3.0.CO;2-O 
George, D. R., Dellasega, C., Whitehead, M. M., \& Bordon, A. (2013). Facebook-based stress management resources for first-year medical students: A multi-method evaluation. Computers in Human Behavior, 29(3), 559562. https://doi.org/10.1016/j.chb.2012.12.008

Greenberg, J. S. (1981). A study of stressors in the college student population. Health Education, 12(4), 8-12. https://doi.org/10.1080/00970050.1981.10616807

Griffiths, M. D. (2019). The evolution of the 'components model of addiction' and the need for a confirmatory approach in conceptualizing behavioral addictions. Dusunen Adam: The Journal of Psychiatry and Neurological Sciences, 32(3), 179-184. https://doi.org/10.14744/DAJPNS.2019.00027

Gutiérrez, J. D.-S., de Fonseca, F. R., \& Rubio, G. (2016). Cell-phone addiction: A review. Frontiers in Psychiatry, 7, Article 175. https://doi.org/10.3389/fpsyt.2016.00175

Hammen, C. (2005). Stress and depression. Annual Review of Clinical Psychology, 1, 293-319. https://doi.org/10.1146/annurev.clinpsy.1.102803.143938

Hayes, A. F. (2017). Introduction to mediation, moderation, and conditional process analysis: A regression-based approach (2nd ed.). Guilford Press.

Johnson, B. K., \& Knobloch-Westerwick, S. (2014). Glancing up or down: Mood management and selective social comparisons on social networking sites. Computers in Human Behavior, 41, 33-39.

https://doi.org/10.1016/j.chb.2014.09.009

Jun, S., \& Choi, E. (2015). Academic stress and Internet addiction from general strain theory framework. Computers in Human Behavior, 49, 282-287. https://doi.org/10.1016/j.chb.2015.03.001

Kandel, D. B., Raveis, V. H., \& Davies, M. (1991). Suicidal ideation in adolescence: Depression, substance use, and other risk factors. Journal of Youth and Adolescence, 20(2), 289-309. https://doi.org/10.1007/BF01537613

Kardefelt-Winther, D. (2014a). A conceptual and methodological critique of internet addiction research: Towards a model of compensatory internet use. Computers in Human Behavior, 31(1), 351-354.

https://doi.org/10.1016/j.chb.2013.10.059

Kardefelt-Winther, D. (2014b). The moderating role of psychosocial well-being on the relationship between escapism and excessive online gaming. Computers in Human Behavior, 38, 68-74.

https://doi.org/10.1016/j.chb.2014.05.020

Kim, D., Lee, Y., Lee, J., Nam, J. K., \& Chung, Y. (2014). Development of Korean Smartphone Addiction Proneness Scale for youth. PLOS ONE, 9(5), Article e97920. https://doi.org/10.1371/journal.pone.0097920

Kim, Y., Wang, Y., \& Oh, J. (2016). Digital media use and social engagement: How social media and smartphone use influence social activities of college students. Cyberpsychology, Behavior, and Social Networking, 19(4), 264269. https://doi.org/10.1089/cyber.2015.0408

Kreek, M. J., Nielsen, D. A., Butelman, E. R., \& LaForge, K. S. (2005). Genetic influences on impulsivity, risk taking, stress responsivity and vulnerability to drug abuse and addiction. Nature Neuroscience, 8(11), 1450-1457. https://doi.org/10.1038/nn1583

Kwon, M., Lee, J., Won, W.-Y., Park, J.-W., Min, J.-A., Hahn, C., Gu, X., Choi, J.-H., \& Kim, D.-J. (2013). Development and validation of a Smartphone Addiction Scale (SAS). PLOS ONE, 8(2), Article e56936.

https://doi.org/10.1371/journal.pone.0056936 
Lapierre, M. A., Zhao, P., \& Custer, B. E. (2019). Short-term longitudinal relationships between smartphone use/dependency and psychological well-being among late adolescents. Journal of Adolescent Health, 65(5), 607612. https://doi.org/10.1016/j.jadohealth.2019.06.001

Lazarus, R. S. (1966). Psychological stress and the coping process. McGraw-Hill.

Lazarus, R. S., \& Folkman, S. (1984). Stress, appraisal, and coping. Springer.

Lee, H., Ahn, H., Nguyen, T. G., Choi, S.-W., \& Kim, D. J. (2017). Comparing the self-report and measured smartphone usage of college students: A pilot study. Psychiatry Investigation, 14(2), 198-204.

https://doi.org/10.4306/pi.2017.14.2.198

Leung, L. (2007). Stressful life events, motives for Internet use, and social support among digital kids. CyberPsychology \& Behavior, 10(2), 204-215. https://doi.org/10.1089/cpb.2006.9967

Lewin, J. E., \& Sager, J. K. (2008). Salesperson burnout: A test of the coping-mediational model of social support. Journal of Personal Selling \& Sales Management, 28(3), 233-246. https://doi.org/10.2753/PSS0885-3134280302

Li, D., Li, X., Wang, Y., Zhao, L., Bao, Z., \& Wen, F. (2013). School connectedness and problematic Internet use in adolescents: A moderated mediation model of deviant peer affiliation and self-control. Journal of Abnormal Child Psychology, 41(8), 1231-1242. https://doi.org/10.1007/s10802-013-9761-9

Li, D., Zhang, W., Li, X., Zhen, S., \& Wang, Y. (2010). Stressful life events and problematic Internet use by adolescent females and males: A mediated moderation model. Computers in Human Behavior, 26(5), 1199-1207. https://doi.org/10.1016/j.chb.2010.03.031

Mayhew, R., \& Edelmann, R. J. (1989). Self-esteem, irrational beliefs and coping strategies in relation to eating problems in a non-clinical population. Personality and Individual Differences, 10(5), 581-584.

https://doi.org/10.1016/0191-8869(89)90042-1

Muusses, L. D., Finkenauer, C., Kerkhof, P., \& Billedo, C. J. (2014). A longitudinal study of the association between compulsive Internet use and wellbeing. Computers in Human Behavior, 36, 21-28.

https://doi.org/10.1016/j.chb.2014.03.035

Pivetta, E., Harkin, L., Billieux, J., Kanjo, E., \& Kuss, D. J. (2019). Problematic smartphone use: An empirically validated model. Computers in Human Behavior, 100, 105-117. https://doi.org/10.1016/j.chb.2019.06.013

Radloff, L. S. (1977). The CES-D scale: A self-report depression scale for research in the general population. Applied Psychological Measurment, 1(3), 385-401. https://doi.org/10.1177/014662167700100306

Rotondi, V., Stanca, L., \& Tomasuolo, M. (2017). Connecting alone: Smartphone use, quality of social interactions and well-being. Journal of Economic Psychology, 63, 17-26. https://doi.org/10.1016/j.joep.2017.09.001

Samaha, M., \& Hawi, N. S. (2016). Relationships among smartphone addiction, stress, academic performance, and satisfaction with life. Computers in Human Behavior, 57, 321-325.

https://doi.org/http://dx.doi.org/10.1016/j.chb.2015.12.045

Takizawa, T., Kondo, T., Sakihara, S., Ariizumi, M., Watanabe, N., \& Oyama, H. (2006). Stress buffering effects of social support on depressive symptoms in middle age: Reciprocity and community mental health. Psychiatry and Clinical Neurosciences, 60(6), 652-661. https://doi.org/10.1111/j.1440-1819.2006.01579.x

Taylor, K., \& Silver, L. (2019). Smartphone ownership is growing rapidly around the world, but not always equally. Pew Research Center. https://www.pewglobal.org/2019/02/05/smartphone-ownership-is-growing-rapidly-around-theworld-but-not-always-equally/ 
Tokunaga, R. S. (2014). A unique problem or the manifestation of a preexisting disorder? The mediating role of problematic internet use in the relationships between psychosocial problems and functional impairment. Communication Research, 41(4), 531-560. https://doi.org/10.1177/0093650212450910

Tsai, H. F., Cheng, S. H., Yeh, T. L., Shih, C.-C., Chen, K. C., Yang, Y. C., \& Yang, Y. K. (2009). The risk factors of Internet addiction - A survey of university freshmen. Psychiatry Research, 167(3), 294-299.

https://doi.org/10.1016/j.psychres.2008.01.015

Van Deursen, A. J. A. M., Bolle, C. L., Hegner, S. M., \& Kommers, P. A. M. (2015). Modeling habitual and addictive smartphone behavior: The role of smartphone usage types, emotional intelligence, social stress, self-regulation, age, and gender. Computers in Human Behavior, 45, 411-420. https://doi.org/10.1016/j.chb.2014.12.039

Van Praag, H. M. (2004). Can stress cause depression? Progress in Neuro-Psychopharmacology and Biological Psychiatry, 28(5), 891-907. https://doi.org/10.1016/j.pnpbp.2004.05.031

Wichianson, J. R., Bughi, S. A., Unger, J. B., Spruijt-Metz, D., \& Nguyen-Rodriquez, S. T. (2009). Perceived stress, coping and night-eating in college students. Stress and Health, 25(3), 235-240. https://doi.org/10.1002/smi.1242

Yao, M. Z., \& Zhong, Z.-j. (2014). Loneliness, social contacts and Internet addiction: A cross-lagged panel study. Computers in Human Behavior, 30, 164-170. https://doi.org/10.1016/j.chb.2013.08.007 


\section{Correspondence to:}

Matthew A. Lapierre

University of Arizona

Department of Communication

Communication Building, Room 211

1103 E. University Blvd

Tucson, AZ 85721

USA

Email: mlapierre(at)email.arizona.edu

Editorial record: First submission received on September 4, 2019. Revisions received on May 1, 2020, August 21, 2020 and November 5, 2020. Accepted for publication on November 9, 2020.

Editor in charge: David Smahel

\section{About Authors}

Pengfei Zhao (M.A., University of Arizona) is a Ph.D. student in the field of communication at Cornell University. Her research interests lie at the intersection of new communication technology, interpersonal communication, and well-being. Specifically, she's interested in studying the effects of social media and smartphones on selfdisclosure, relationship development, and mental health and well-being.

Matthew A. Lapierre (Ph.D., University of Pennsylvania) is an Assistant Professor of Communication at the University of Arizona. His research explores how media affects health and well-being, particularly among children/families. His research projects include investigations of the influence of marketing cues on children's consumer behavior, the development of children's persuasion awareness/susceptibility, how media engagement influences gun attitudes among adults and children, and the role smartphones play in shaping our interpersonal relationships and well-being. 